\title{
EVALUATING APPROXIMATIONS TO THE OPTIMAL EXERCISE BOUNDARY FOR AMERICAN OPTIONS
}

\author{
ROLAND MALLIER
}

Received 24 March 2001 and in revised form 5 October 2001

We consider series solutions for the location of the optimal exercise boundary of an American option close to expiry. By using Monte Carlo methods, we compute the expected value of an option if the holder uses the approximate location given by such a series as his exercise strategy, and compare this value to the actual value of the option. This gives an alternative method to evaluate approximations. We find the series solution for the call performs excellently under this criterion, even for large times, while the asymptotic approximation for the put is very good near to expiry but not so good further from expiry.

\section{Introduction}

Options are derivative financial instruments giving the holder the right but not the obligation to buy (or sell) an underlying asset. They have numerous uses, such as speculation, hedging, generating income, and they contribute to market completeness. Although options have existed for much longer, their use has become much more widespread since 1973 when two of the most significant events in the history of options occurred. The first of these was the publication of the Black-Scholes option pricing formula (Black and Scholes [10]), which enabled investors to price certain options, and which Merton [46] extended to include a continuous dividend yield. The second important event was the opening of the Chicago Board Options Exchange (CBOE), which was really the first secondary market for options. Before the CBOE opened its doors, it was extremely difficult for an investor to sell any options that he might 
own, so that he was left with the choice of holding the option to expiry, or exercising early if that was permitted. With the advent of the $\mathrm{CBOE}$, he had the additional choice of reselling the options to another investor.

There are various ways of categorizing options, one method being by the exercise characteristics. Options are usually either European, meaning they can be exercised only at expiry, which is a pre-determined date specified in the option contract, or American, meaning they can be exercised at or before expiry, at the holder's discretion. A third, less common, type is Bermudan, which can be exercised early, but only on a finite number of pre-specified occasions. European options are fairly easy to value. However, American options are much harder since because they can be exercised early, the holder must decide whether and when to exercise such an option, and this is one of the best-known problems in mathematical finance, leading to an optimal exercise boundary and an optimal exercise policy, which if followed will maximize the expected return. Ideally, an investor would be able to constantly calculate the expected return from continuing to hold the option, and if that is less than the return from immediate exercise, he should exercise the option. This process would tell the investor the location of the optimal exercise boundary. However, to date no closed form solutions are known for the location of the optimal exercise boundary, except for one or two very special cases. One such special case is the American call with no dividends, when exercise is never optimal, so that the value of the option is the same as that of a European call; indeed, the value of an American call will differ from that of the European only if there is a dividend of sufficient size to make early exercise worthwhile. Another special case is the Roll-Geske-Whaley formula (Roll [48]; Geske [31, 32]; Whaley [52]) for the American call with discrete dividends.

For American options, an investor is primarily concerned with two aspects of the pricing problem: firstly, the location of the optimal exercise boundary, so that he knows whether or not to exercise the option, and secondly, the value of the option. For those cases where exact solutions are not known, it is fairly straightforward to solve the problem numerically, or use one of the numerous approximate solutions and series solutions which appear in the literature; given the importance of American options, it is hardly surprising that a number of different approximations have been proposed over the years to tackle the problem, and a full review of these is beyond the scope of the present study, but we will mention some of the more important ones.

Amongst numerical techniques, the more popular methods include backward recursion methods, such as binomial and trinomial trees (Cox et al. [27]; Boyle [14]) both of which involve integrating the underlying 
stochastic differential equation (DE) for the price of the underlying $S$

$$
d S=\left(r-D_{0}\right) S d t+\sigma S d X,
$$

backwards in time from expiry. In this equation, $r$ is the risk free rate, and $\sigma$ is the volatility and $D_{0}$ the dividend yield of the underlying stock, all of which are taken to be constants in the present study, $d X$ describes the random walk, and $d t$ is the step size. Black and Scholes [10] derived this equation in the absence of dividends, and Merton [46] added the effect of a constant dividend yield. While the assumption of a constant dividend yield is questionable for an option on a single security, it is justifiable for other options, such as foreign exchange, index options, and options on commodities. Finite-difference methods (Brennan and Schwartz [16]; $\mathrm{Wu}$ and Kwok [54]; Wilmott [53]) are also popular, and they involve solving the PDE formulation of the problem for the value $V(S, t)$ of the option (Black and Scholes [10]; Merton [46]),

$$
\frac{\partial V}{\partial t}+\frac{\sigma^{2} S^{2}}{2} \frac{\partial^{2} V}{\partial S^{2}}+\left(r-D_{0}\right) S \frac{\partial V}{\partial S}-r=0,
$$

on a discrete grid. This PDE can be derived by applying a no-arbitrage argument to the stochastic DE (1.1). Geske and Shastri [34] gave an early comparison of finite-difference and binomial tree methods, although of course the state-of-the-art in both methods has come a long way since that study.

Turning to approximate solutions, in the present study, we are evaluating series solutions (in time) for the optimal exercise boundary about expiry, which were first presented by Barles et al. [6] for the American put and Dewynne et al. [28] for the call; we will discuss this approximation in more detail later, but first, we should mention some of the other approximations that have been suggested, the majority of which are very good. One popular approach comprises approximating the equations obeyed by an American option, and then solving these equations exactly. An example of this is the quadratic approximation method used by MacMillan [41] for the valuation of an American put on a nondividend paying stock, which was extended to stocks with dividends by Barone-Adesi and Whaley [8], Barone-Adesi and Elliott [7], and Allegretto et al. [3]. This particular approximation, which involved solving an approximate PDE for the early exercise premium, that is, the amount by which the value of an American exceeds a European, is very popular amongst institutional investors. A second approach involves an integral representation of the early exercise premium, and examples of this include the studies of Carr et al. [23] and Huang et al. [35]. Huang et al.'s method involved recursive computation of the optimal exercise 


\section{Approximations for American options}

boundary by estimating the boundary at only a few points and then using Richardson extrapolation; one advantage claimed by the authors for this method is that it can readily be adapted to a wide variety of American style options in addition to plain vanilla put and calls. Another well-known approximation is the Geske-Johnson formula (Johnson [37]; Geske and Johnson [33]; Bunch and Johnson [19]; Blomeyer [11]) for the American put, which views an American option as a sequence of Bermudan options with the number of exercise dates increasing. Selby and Hodges [49] give an overview of the Roll-Geske-Whaley and GeskeJohnson formulae together with a complete analysis of American call options with an arbitrary number of (discrete) dividends and a suggestion as to how to improve the numerical implementation of the GeskeJohnson formula; a review of the current state-of-the-art of the computational aspects of this problem is given in Gao et al. [30]. Still other approaches that should be mentioned briefly include that of Aitsahlia and Lai $[1,2]$, who have tabulated the values of the options at a number of points and then obtain the values at intermediate points by interpolation, the method of lines of Carr and Faquet [22], the study by Ju [38] in which the optimal exercise boundary was approximated as a multipiece exponential function, and that by Bjerksund and Stensland [9] in which a flat exercise boundary was assumed. One recent and very promising technique is the LUBA (lower and upper bound approximation) of Broadie and Detemple [17]: although no closed form solution is known for the optimal exercise boundary, it is possible to find very tight upper and lower bounds for this boundary. Broadie and Detemple showed that in addition to the LUBA approximation being very accurate, with an RMS error of $0.02 \%$ in the cases studied, it is also very fast. One nice feature of Broadie and Detemple's study was that they included an extensive comparison between their method and other techniques. Melick and Thomas [45] explored the LUBA approximation further, using it to back a PDF out of observed option prices. A very different approach was taken by Carr [21], who obtained semi-explicit approximations for American options by randomizing the time until maturity and then reducing the variance of this random time to maturity. The objective of the randomization in Carr [21] was to simplify the effect of the passage of time on the value of the option, and indeed this simplification is a common aim of many of the approximations mentioned above. At its extreme, this simplification gives us a perpetual American option, which has no time dependence (Merton [46]; Dewynne et al. [28]; Wilmott [53]), or a quasi-stationary method, such as the quadratic approximation mentioned above, where the unsteady term in the PDE $(\partial V / \partial \tau$, being the derivative of the option value with respect to time) is partially or completely ignored. 


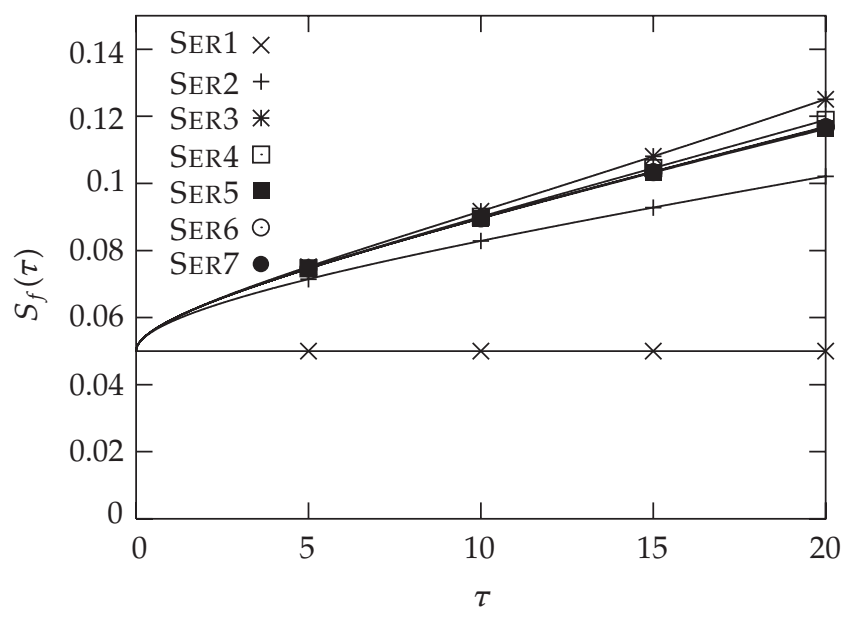

FIGURE 1.1. Exercise boundary for the call from (1.3) for $E=0.9, r=$ $0.05, D_{0}=0.04, \sigma=0.25$. SER1 uses 1 term in the series, etc.

Rather than ignore or simplify the effect of time, the approximation considered in the present study is based on a series in time for the location of the optimal exercise boundary about expiry. The objective of the present study is not to rederive or extend these series, but rather to evaluate how accurate they are using a different metric to previous studies. These series solutions were originally presented by Barles et al. [6] for the American put and Dewynne et al. [28] for the call. A number of additional studies have recently appeared on these series solutions. For the call, Alobaidi and Mallier [4] extended the earlier result of Dewynne et al. to higher order, giving the series as far as the coefficient of the $\tau^{3}$ term,

$$
x_{f}(\tau) \sim a_{0}+a_{1} \tau^{1 / 2}+a_{2} \tau+a_{3} \tau^{3 / 2}+a_{4} \tau^{2}+a_{5} \tau^{5 / 2}+a_{6} \tau^{3}+\cdots,
$$

where $S_{f}=E e^{x_{f}}$ is the location of the optimal exercise boundary ( $E$ being the exercise price of the option) and $\tau=T-t$ is the time remaining to expiry. Series (1.3) is a power series in the time remaining until expiry, with the coefficients $a_{n}$ as far as $n=6$ given in Alobaidi and Mallier [4], the first two coefficients ( $a_{0}$ and $a_{1}$ ) having previously been given by Dewynne et al. and also in several recent texts such as Wilmott [53]. These coefficients are functions of $E, r, \sigma$, and $D_{0}$. The first coefficient, $a_{0}$ gives the location of the free boundary at expiry and can be found by considering the behaviour of the Black-Scholes-Merton PDE at expiry, and the remaining coefficients were derived using a local analysis of the PDE (1.2) close to expiry, which involved rescaling the PDE; more details of the derivation of the series can be found in the references cited 
above. In Figure 1.1, we show the location of the exercise boundary calculated from this series for the call, using the parameters for the first run for the call discussed later in the results section. Apart from the constant boundary (labelled SERI in the figure), the behaviour of the boundary appears similar regardless of the number of terms taken. This happens because the coefficients of the higher order terms are extremely small, so even for $\tau=20$, the higher order terms are fairly unimportant. We should also mention that Dewynne et al. and Alobaidi and Mallier [4] also give a series solution for the value of the option itself, in addition to the location of the optimal exercise boundary which is discussed here; this series solution for the value of the option is a local solution about the position of the free boundary at expiry and depends on both the time remaining until expiry and the distance from the free boundary. For the put, several authors (Kuske and Keller [39]; Stamicar et al. [50]; Bunch and Johnson [20]; Alobaidi and Mallier [5], Evans et al. [29]; Chen and Chadam [24]; Chen et al. [25]) have recently revisited the problem and independently rederived the result of Barles et al. using various techniques; for example, Kuske and Keller used a Green's function to derive an integral equation which they solved iteratively and Alobaidi and Mallier [5] attempted a local analysis of the Black-Scholes-Merton PDE close to expiry, along the lines of the study by Dewynne et al. for the call. For the put, the series is of the form (Evans et al. [29])

$$
S_{f}(\tau) \sim E\left[1-\sigma \sqrt{\tau \log \left(\frac{\sigma^{2}}{8 \pi \tau\left(r-D_{0}\right)^{2}}\right)}\right] .
$$

As with the call, the coefficients in this series are functions of $E, r, \sigma$, and $D_{0}$. It should be noted that this series contains logs, and we should mention that differing values of the coefficient of the log term in (1.4) were given in the various studies mentioned above, which the present author finds somewhat disconcerting; we have used the form given by Evans et al. [29] which appears to be the current consensus. In Figure 1.2, we show the location of the exercise boundary calculated from (1.4) for the put, using the parameters for the first run for the put discussed later in the results section. It is interesting to note that, after initially decreasing with increasing $\tau$, the boundary begins to increase once again. Obviously, this behaviour is unphysical, and the actual boundary slopes downwards.

Strictly speaking, series (1.3) and (1.4) are derived in the limit $\tau \rightarrow 0$, that is close to expiry, although we will be using them for larger values of $\tau$ as well. Both of these series are valid for $0 \leq D_{0}<r$; the results for $D_{0}>$ $r$ can be written down fairly easily using the put-call symmetry condition of Chesney and Gibson [26] and McDonald and Schroder [44], namely 


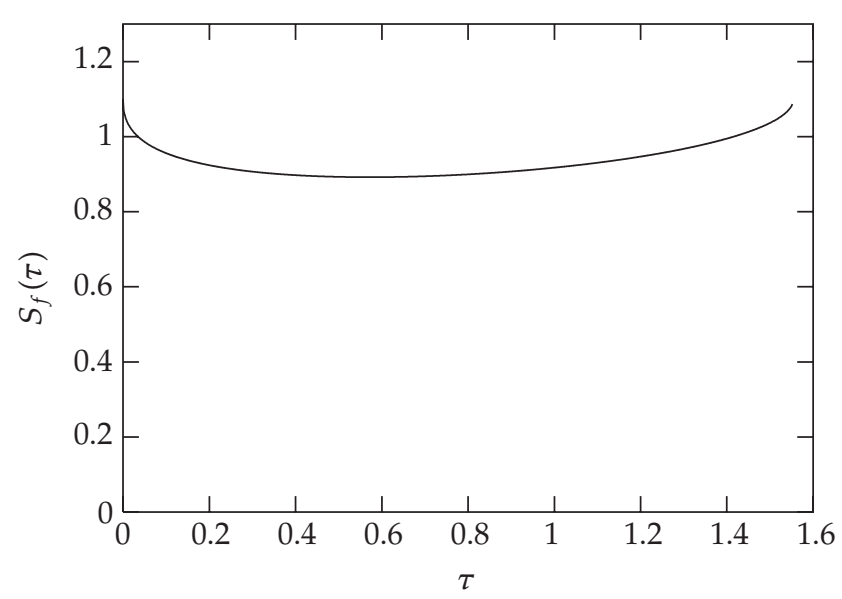

FIGURE 1.2. Exercise boundary for the put from (1.4) for $E=1.1, r=$ $0.05, D_{0}=0.01$, and $\sigma=0.25$.

that the prices of the call and put are related by

$$
C\left[S, E, D_{0}, r\right]=P\left[E, S, r, D_{0}\right],
$$

while the positions of the optimal exercise boundary for the call and put are related by

$$
S_{f}^{c}\left[t, E, r, D_{0}\right]=\frac{E^{2}}{S_{f}^{p}\left[t, E, D_{0}, r\right]} .
$$

Several of the above authors have explored how good these series are, but the approach taken has always been to examine how far the approximate boundary is located from the exercise boundary calculated by some other means. An example of this is the recent study by Stamicar et al. [50], who compared the location of the free boundary for the American put obtained using series (1.4) to the location obtained using other methods: a (1000 step) binomial tree, the (numerical) solution of an integral equation and the quadratic approximation; although this comparison was only carried out for extremely small times $(\tau \leq 0.05)$, agreement between (1.4) and the other methods was only as good as the third significant figure at $\tau=0.05$. We would suggest than an alternative approach would be to compare the expected returns that an investor would receive if he used these asymptotic solutions with the actual value of the option. Our approach is perhaps more valuable to a real-life investor, who would be happy if the approximate boundary was far from the real boundary but his expected returns were very close to the value of the 
option, but considerably less so if the two boundaries were very close but the expected returns were much less than the value of the option.

We use Monte Carlo simulation to tackle the valuation of the option when series (1.3) and (1.4) are used as exercise boundaries. This approach is well-suited for this particular problem, since the underlying stock price $S$ is assumed to follow a random walk. The use of Monte Carlo methods for option pricing was pioneered by Boyle [13], and these methods have since become extremely popular because they are both powerful and extremely flexible. Although the use of Monte Carlo methods to value American options is still a nebulous problem, with for example several researchers pursuing Malliavin calculus while others are attempting different approaches (e.g., Tilley [51]; Bossaert [12]; Broadie and Glasserman [18]; Ibanez and Zapatero [36]; Boyle et al. [15]; and Mallier [42]), these difficulties stem from the need to locate the optimal exercise boundary, and for the problem studied here, that is not an issue, rather, we are calculating what an option is worth if a strategy based on the location of the boundary given by the asymptotics is followed, and so the location of our exercise boundary is already known. Returning to option pricing in general, in this context, Monte Carlo methods involve the direct stochastic integration of the underlying Langevin equation (1.1) for the stock price, which is assumed to follow a log-normal random walk or geometric Brownian motion. The heart of any Monte Carlo method is the random number generator, and our code employed the Netlib routine RANLIB, which produces random numbers which are uniformly distributed on the range $(0,1)$ and which were then converted to normally distributed random numbers. This routine was itself based on the article by L'Ecuyer and Cote [40]. Antithetic variables were used to speed convergence, and a large number of realizations $(100,000)$ were performed to ensure accurate results. Our simulations, including other runs not presented here, were performed on the Beowulf cluster at the University of Western Ontario. Our Monte Carlo code has been used by us previously for other work (Mallier and Alobaidi [43]; Mallier [42]). We took a fairly small step size $(d t=0.0001)$ for accuracy reasons. Typically, the stochastic DE (1.1) is integrated numerically, and then the option valued by calculating the payoff, which is $\max [S-E, 0]$ in the case of a vanilla call and $\max [E-S, 0]$ in the case of a vanilla put. One point worth mentioning is that the equation is the same for both a put and a call, so that, as observed by Merton [47], it is the boundary conditions that distinguish options. Returning to the Monte Carlo simulation of (1.1), if we assume that the simulation is started at time $t_{0}$ and ends at expiry $T$, then the other parameters which affect the simulations are the initial stock price $S_{0}=S\left(t_{0}\right)$, the exercise price $E$ and the initial time to expiry, $\tau_{0}=T-t_{0}$. For each realization, at each time step, 
we first check to see if the exercise criteria has been satisfied, and either exercise at that step or continue to the next time step, and repeat this procedure either the option has been exercised or we reach expiry, at which time the option is either exercised or expires worthless. For each realization, we calculate the payoff, $\max \left[S\left(T_{E}\right)-E, 0\right]$ for the call and $\max \left[E-S\left(T_{E}\right), 0\right]$ for the put, where $T_{E}$ is the time at which the option was exercised. We then discount this value back to the starting time to find its present value. The value of the option is the average over all realizations of this present value. In Section 2, we present our numerical results, and give the value of the options if the series solutions (1.3) and (1.4) are used as an exercise strategy. For comparison purposes, we also give the value of both an American and European option calculated using a standard binomial tree, so that the reader can assess how useful the series solutions are. In addition, we compare our results to several of the other approximations mentioned above, specifically the LUBA, GeskeJohnson, Quadratic and Method-of-Lines approximations, again so the reader can assess the usefulness of the series discussed here. These results are discussed further in Section 3.

\section{Results}

In this section, we present Monte Carlo simulations of the stochastic DE (1.1), using a step size of 0.0001 and 100,000 paths in each simulation. The asymptotic solutions for the optimal exercise boundary (1.3) and (1.4) were used as our exercise criteria in these simulations: for the put, if the value of the option was below the exercise boundary (1.4), then the option was exercised with payoff $\max [E-S, 0]$, and similarly for the call, if the option price was above the exercise boundary (1.3), the option was exercised with payoff $\max [S-E, 0]$. For the put, a single set of simulations were performed using (1.4) as the exercise boundary. By contrast, for the call, a series of simulations were performed, using between 1 and 7 terms in series (1.3); when only one term is used, our boundary is simply a horizontal line, while when two terms are used, we are using the solution presented by Dewynne et al. [28]. Alobaidi and Mallier [4] give the coefficients of the first seven terms in the series. We also compare the results obtained using these asymptotic boundaries to the true value of an American option obtained using a standard binomial tree with 100,000 steps; we used such a large number of steps in order to ensure that we had a very accurate reference value to which to compare our results. In one or two of the simulations, the Monte Carlo value is slightly higher than the binomial value: this is an anomaly caused by two things: firstly, although we are using very small step sizes and many paths, there will still be very small numerical errors in 
our computations, and secondly, since with Monte Carlo we only use a finite number of paths, it is possible that the option value using only those paths is slightly higher than the value if every possible path were to be used.

\subsection{The call}

For the call, we present some sample runs in Tables 2.1, 2.2, 2.3, and 2.4. Each table represents a different run, and results are presented for a variety of times $(0.5,1,2.5,5,10$, and 20$)$. In each case, we give the value of both a European and an American call option, and then the value of the option if the series solution (1.3) is used as an exercise strategy. The columns labelled SER1, SER2, and so forth, represent the value if $1,2, \ldots$ terms in the series are used. For the call, with the exception of the column labelled SER1 in Tables 2.1 and 2.3, the results appear to be excellent even for long times such as $\tau_{0}=10$ and 20, and using the series boundary enables us to capture almost all of the early exercise premium of an American call. It is perhaps surprising that the boundary performs so well so far from expiry, since the series was derived in the limit $\tau \rightarrow 0$, but it is also very encouraging. The column labelled SER1 uses a horizontal boundary $\left(S_{f}=E e^{a_{0}}=r E / D_{0}\right)$, so the simulations in that column would be expected to be the least good. Surprisingly, however, for several of the simulations, taking more terms in the series makes the results worse rather than better. Several points should be noted about this: most obviously, series (1.3) was derived for small $\tau$ and will converge to the optimal exercise boundary in that limit as we increase the number of terms. However, convergence in this sense means that the boundary coming from the series physically tends to the optimal exercise boundary. This can be seen from Figure 1.1. If a large enough number of terms were taken in the series, close to expiration it would also converge to the optimal exercise boundary under the dollar metric considered here. From Figure 1.1, we can see that physically, the location of the boundary can be above or below the value to which it is converging, depending on the number of terms used in the series, and it is this behaviour which we suspect is responsible for the behaviour with respect to the dollar metric mentioned above. In addition, clearly series (1.3) must have a finite radius of convergence (in physical space), since we know from the perpetual American call that the optimal exercise boundary tends to a finite value as $\tau \rightarrow \infty$ while our series will blow up as $\tau \rightarrow \infty$. We would claim however that Figure 1.1 suggests that we are inside that radius of convergence for the cases studied here.

Over a much larger sampling of results (across 100 values), a subset of which is presented graphically in Figure 2.1, we found that, for the call, the average absolute error in the value of the option obtained using 
TABLE 2.1. Call: Run $1 ; S_{0}=0.8, E=0.9, r=0.05, D_{0}=0.04, \sigma=0.25$. Euro. and AMER. are values of European and American options computed using a 100,000 step binomial tree. Remaining columns are the values of an American option computed using a 100,000 path Monte Carlo simulation taking series (1.3) as the exercise boundary (SER1 uses one term in the series, SER2 uses two terms, etc.).

\begin{tabular}{l|llllll}
\hline$\tau_{0}$ & 0.5 & 1 & 2.5 & 5 & 10 & 20 \\
\hline EURO. & 0.023196 & 0.044425 & 0.086952 & 0.127351 & 0.161539 & 0.160889 \\
AMER. & 0.023202 & 0.044504 & 0.087940 & 0.132098 & 0.180190 & 0.219914 \\
SER1 & 0.023408 & 0.043716 & 0.077372 & 0.100222 & 0.116832 & 0.125331 \\
SER2 & 0.023362 & 0.044499 & 0.087299 & 0.130012 & 0.177400 & 0.218152 \\
SER3 & 0.023360 & 0.044484 & 0.087285 & 0.129825 & 0.176785 & 0.217928 \\
SER4 & 0.023361 & 0.044490 & 0.087232 & 0.129666 & 0.176066 & 0.215930 \\
SER5 & 0.023361 & 0.044490 & 0.087232 & 0.129672 & 0.176089 & 0.216186 \\
SER6 & 0.023361 & 0.044490 & 0.087230 & 0.129666 & 0.176168 & 0.216519 \\
SER7 & 0.023361 & 0.044490 & 0.087230 & 0.129666 & 0.176172 & 0.216472 \\
\hline
\end{tabular}

TABLE 2.2. Call: Run 2; as in Table 2.1 but $S_{0}=0.7, E=0.4, r=0.4$, $D_{0}=0.1$, and $\sigma=0.1$.

\begin{tabular}{l|llllll}
\hline$\tau_{0}$ & 0.5 & 1 & 2.5 & 5 & 10 & 20 \\
\hline EURO. & 0.305647 & 0.310618 & 0.321866 & 0.330500 & 0.322075 & 0.260397 \\
AMER. & 0.305647 & 0.310618 & 0.321950 & 0.332401 & 0.339442 & 0.341323 \\
SER1 & 0.305655 & 0.310630 & 0.321950 & 0.332188 & 0.338805 & 0.340347 \\
SER2 & 0.305655 & 0.310630 & 0.321925 & 0.332240 & 0.338710 & 0.338210 \\
SER3 & 0.305655 & 0.310632 & 0.321924 & 0.332318 & 0.339312 & 0.339703 \\
SER4 & 0.305655 & 0.310631 & 0.321921 & 0.332322 & 0.339311 & 0.340906 \\
SER5 & 0.305655 & 0.310631 & 0.321917 & 0.332319 & 0.339364 & 0.341184 \\
SER6 & 0.305655 & 0.310631 & 0.321917 & 0.332318 & 0.339363 & 0.341221 \\
SER7 & 0.305655 & 0.310631 & 0.321917 & 0.332317 & 0.339360 & 0.341285 \\
\hline
\end{tabular}

one term in series (1.3) together with Monte Carlo simulation was $2.97 \%$, and using two to seven terms $0.18 \%$. Over the runs we did, the maximum error in any run using one term in (1.3) together with Monte Carlo was $13.5 \%$, while if we used two to seven terms it was $0.84 \%$. In Figure 2.1 , we show the percentage absolute error against time for a number of runs for the call, showing runs which were done using one, two, four, and seven terms in series (1.3). In the first of these, it can be seen that if we use only one term (i.e., a constant boundary), the error, while fairly small for very small tenor, or time remaining until expiry, increases rapidly with increasing tenor, and by the time we reach $T-t=10$, the percentage error is often fairly large, being about $13 \%$ in several runs, which would clearly be unacceptable in real world applications. By contrast, if we take more terms in the series (and we note that the plots for three, five, and 
82 Approximations for American options

TABLE 2.3. Call: Run 3; as in Table 2.1 but $S_{0}=0.8, E=0.8, r=0.05$, $D_{0}=0.04$, and $\sigma=0.25$.

\begin{tabular}{l|llllll}
\hline$\tau_{0}$ & 0.5 & 1 & 2.5 & 5 & 10 & 20 \\
\hline EURO. & 0.057068 & 0.079963 & 0.121167 & 0.157192 & 0.183686 & 0.173108 \\
AMER. & 0.057105 & 0.080202 & 0.122984 & 0.164248 & 0.207828 & 0.242927 \\
SER1 & 0.056832 & 0.077295 & 0.104736 & 0.120718 & 0.131258 & 0.136862 \\
SER2 & 0.057231 & 0.080076 & 0.121674 & 0.161699 & 0.204715 & 0.241911 \\
SER3 & 0.057231 & 0.080064 & 0.121495 & 0.161352 & 0.203957 & 0.241179 \\
SER4 & 0.057230 & 0.080054 & 0.121478 & 0.161038 & 0.202717 & 0.238469 \\
SER5 & 0.057230 & 0.080054 & 0.121478 & 0.161066 & 0.202783 & 0.238703 \\
SER6 & 0.057230 & 0.080054 & 0.121479 & 0.161078 & 0.202828 & 0.239001 \\
SER7 & 0.057230 & 0.080054 & 0.121479 & 0.161076 & 0.202825 & 0.238997 \\
\hline
\end{tabular}

TABle 2.4. Call: Run 4; as in Table 2.1 but $S_{0}=0.8, E=0.6, r=0.1$, $D_{0}=0.08$, and $\sigma=0.1$.

\begin{tabular}{l|llllll}
\hline$\tau_{0}$ & 0.5 & 1 & 2.5 & 5 & 10 & 20 \\
\hline EURO. & 0.197894 & 0.195609 & 0.188215 & 0.173994 & 0.141084 & 0.081668 \\
AMER. & 0.2 & 0.2 & 0.200445 & 0.202105 & 0.204393 & 0.205797 \\
SER1 & 0.2 & 0.2 & 0.2 & 0.2 & 0.2 & 0.2 \\
SER2 & 0.2 & 0.2 & 0.200509 & 0.201784 & 0.202807 & 0.198361 \\
SER3 & 0.2 & 0.2 & 0.200491 & 0.202009 & 0.204418 & 0.205653 \\
SER4 & 0.2 & 0.2 & 0.200538 & 0.201945 & 0.203979 & 0.203402 \\
SER5 & 0.2 & 0.2 & 0.200531 & 0.201988 & 0.204259 & 0.205781 \\
SER6 & 0.2 & 0.2 & 0.20052758 & 0.201997804 & 0.204316949 & 0.205777624 \\
SER7 & 0.2 & 0.2 & 0.200526 & 0.202003 & 0.204302 & 0.205820 \\
\hline
\end{tabular}

six terms, which are not presented here, are similar to those for two, four, and seven terms), the percentage error does not appear to depend that much on tenor, and even for times as large as $T-t=10$, this error remains well under $1 \%$ for the runs studied here. As a point of comparison for the accuracy of these results, in real life, option prices trade in discrete increments (the tick size). On the CBOE, for example, the minimum tick size for DJIA options trading below $\$ 300$ is $\$ 5$, and $\$ 10$ for those above $\$ 300$, while for equity options, the minimum tick size for options trading below $\$ 300$ is $\$ 6.25$, and $\$ 12.50$ for those above $\$ 300$, so that for an equity option trading below $\$ 300$, the tick size is in excess of $2 \%$, meaning that the loss in value from using series (1.3) as the basis of an exercise strategy is considerably smaller than the tick size.

In addition, in Tables 2.5 and 2.6, our results for the value of an option obtained using the series solution combined with Monte Carlo simulation are compared to previously published values obtained using other methods, specifically the LUBA approximation, the 2-point Geske- 
TABLE 2.5. Call: Comparison with other methods; $\tau=0.5, E=100$, $r=0.07, D_{0}=0.03, \sigma=0.3$. Euro. and AMER. are values of European and American options computed using a 100,000 step binomial tree. SER7 is the value of an American option computed using a 100,000 path Monte Carlo simulation taking 7 terms in series (1.3) as the exercise boundary. LUBA is the LUBA approximation (Broadie and Detemple [17]). GJ is the (2-point) Geske-Johnson approximation (Geske and Johnson [33]). QUAD. is the quadratic approximation (MacMillan [41]; Barone-Adesi and Whaley [8]). ML is the method of lines based on $n=3$ time steps (Ju [38]).

\begin{tabular}{l|lllll}
\hline$S_{0}$ & 80 & 90 & 100 & 110 & 120 \\
\hline EURO. & 1.664 & 4.495 & 9.251 & 15.797 & 23.706 \\
AMER. & 1.664 & 4.495 & 9.251 & 15.797 & 23.706 \\
SER7 & 1.658 & 4.475 & 9.23 & 15.773 & 23.688 \\
LUBA & 1.664 & 4.495 & 9.251 & 15.798 & 23.706 \\
GJ & 1.664 & 4.495 & 9.251 & 15.798 & 23.706 \\
QUAD. & 1.665 & 4.495 & 9.251 & 15.799 & 23.709 \\
ML & 1.663 & 4.500 & 9.284 & 15.845 & 23.774 \\
\hline
\end{tabular}

TABle 2.6. Call: Comparison with other methods; as in Table 2.5 but $\tau=3.0$.

\begin{tabular}{l|lllll}
\hline$S_{0}$ & 80 & 90 & 100 & 110 & 120 \\
\hline EURO. & 12.132 & 17.343 & 23.301 & 29.882 & 36.972 \\
AMER. & 12.145 & 17.368 & 23.348 & 29.963 & 37.103 \\
SER7 & 12.179 & 17.405 & 23.394 & 29.983 & 37.136 \\
LUBA & 12.167 & 17.368 & 23.383 & 30.001 & 37.142 \\
GJ & 12.137 & 17.355 & 23.331 & 29.946 & 37.091 \\
QUAD. & 12.282 & 17.553 & 23.586 & 30.259 & 37.459 \\
ML & 12.137 & 17.391 & 23.380 & 30.009 & 37.146 \\
\hline
\end{tabular}

Johnson approximation, the quadratic approximation and the method of lines. The parameter values chosen are the same as those used by Broadie and Detemple [17] for their comparison of their LUBA approximation to other methods. On the basis of these tables, while we would not claim that the series approximation (1.3) studied here is necessarily more accurate than the other approximations included in the tables, it is clearly competitive with those other approximations in terms of accuracy, and given our comments on the tick sizes for equity options above, the results provided by series (1.3) coupled with Monte Carlo would be more than accurate enough for an investor in the real world. Where we would claim series (1.3) has an advantage over many of the other approximations is in its ease of calculation: it could literally be 
84 Approximations for American options

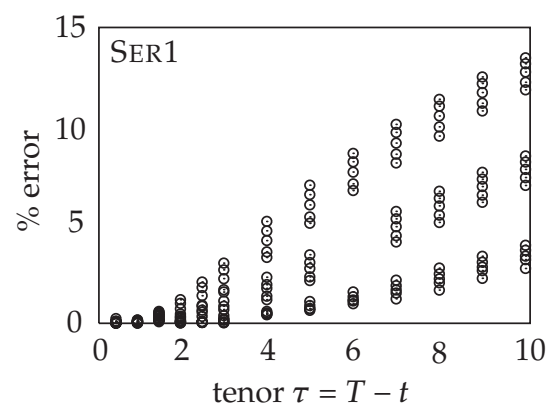

(a)

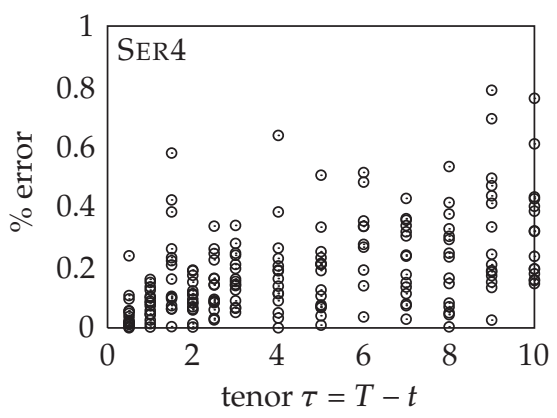

(c)

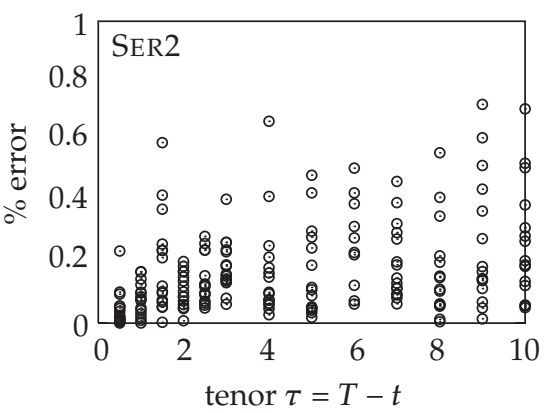

(b)

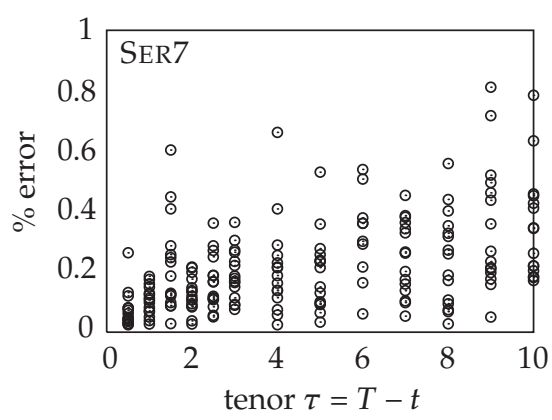

(d)

FIgURE 2.1. Percentage error in the value of a call option using series (1.3) for a number of sample runs. (a) 1 term in the series; (b) 2 terms; (c) 4 terms; (d) 7 terms.

programmed into a financial calculator. We would claim that this combination of ease of calculation combined with acceptable accuracy should make it an attractive method to investors.

\subsection{The put}

In Tables 2.7, 2.8, 2.9, and 2.10, we show some sample results for the put. Three values of the option are shown for each time: the European value and the American value, both of which were computed using a binomial tree, and the series value, that is the value using the approximation (1.4) as the exercise boundary. It should be noted that because of the logarithm inside the square root, this approximation is only valid for $\tau \leq \sigma^{2} /\left(8 \pi\left(r-D_{0}\right)^{2}\right)$; for each of the runs presented here, the largest time for which results are presented (e.g., 1.5442 in Table 2.7) is very 
TABle 2.7. Put: Run 1; $S_{0}=1.0, E=1.1, r=0.05, D_{0}=0.01, \sigma=0.25$. Euro. and AMER. are values of European and American options computed using a 100,000 step binomial tree. SER is the value of an American option computed using a 100,000 path Monte Carlo simulation taking series (1.4) as the exercise boundary.

\begin{tabular}{l|lll}
\hline$\tau_{0}$ & 0.5 & 1 & 1.5542 \\
\hline EURO. & 0.118224 & 0.131892 & 0.141266 \\
AMER. & 0.123107 & 0.140754 & 0.154635 \\
SER. & 0.122967 & 0.136486 & 0.1 \\
\hline
\end{tabular}

TABLE 2.8. Put: Run 2; as in Table 2.7 but $S_{0}=1.0, E=1.0, r=0.1$, $D_{0}=0.04$, and $\sigma=0.25$.

\begin{tabular}{l|ll}
\hline$\tau_{0}$ & 0.5 & 0.6907 \\
\hline EURO. & 0.054505 & 0.060662 \\
AMER. & 0.057783 & 0.065476 \\
SER. & 0.056123 & 0.023997 \\
\hline
\end{tabular}

close to this value. We see that for times very close to expiry, the approximation is very good, but for more distant times, it is fairly poor. For example, in Tables 2.7 and 2.9, the asymptotic boundary captures almost the entire American value when $\tau=0.5$, while for that same time, the asymptotic boundary and the optimal exercise boundary in Table 2.10 both lead to immediate exercise so that both have the same value. The results for $\tau=0.5$ in Table 2.8 are not quite as good: for this particular run, the asymptotic boundary captures only $47 \%$ of the early exercise premium, that is the difference between the European value and the American value. As we get further away from expiry, the results for the asymptotics become less good, and in two of the four cases shown, the value of the option using the asymptotics is actually less than the European value for larger times. It would appear then that the asymptotic boundary for the put is only really useful very close to expiry; paradoxically, this is the region where the difference in value between the American and European options tends to be smallest close to expiry, and knowledge of the optimal exercise boundary is least useful: an investor holding an option until expiry in this region will lose very little compared to one who follows the optimal exercise policy.

As with the call, we compared results using series (1.4) to results obtained using a 100,000 step binomial tree over a much larger sampling of options. We found that, for the put, if we used both the constant and the log term in series (1.4), the results were reasonably good for small times, but very poor for larger times. If we used only the constant term, 
TABle 2.9. Put: Run 3; as in Table 2.7 but $S_{0}=4.0, E=4.0, r=0.2$, $D_{0}=0.16$, and $\sigma=0.25$.

\begin{tabular}{l|lll}
\hline$\tau_{0}$ & 0.5 & 1 & 1.5542 \\
\hline EURO. & 0.222570 & 0.269814 & 0.287994 \\
AMER. & 0.236074 & 0.303693 & 0.347739 \\
SER. & 0.236583 & 0.302210 & 0.115171 \\
\hline
\end{tabular}

TABle 2.10. Put: Run 4; as in Table 2.7 but $S_{0}=0.9, E=1.2, r=0.5$, $D_{0}=0.02$, and $\sigma=0.25$.

\begin{tabular}{l|llll}
\hline$\tau_{0}$ & 0.5 & 1 & 2.5 & 2.7631 \\
\hline EURO. & 0.284112 & 0.278906 & 0.272305 & 0.271114 \\
AMER. & 0.3 & 0.301452 & 0.313596 & 0.315639 \\
SER. & 0.3 & 0.300236 & 0.3 & 0.3 \\
\hline
\end{tabular}

the results are extremely poor even for small times. For $\tau=0.5$, we found that average absolute error in the value of the option obtained using one term (the constant term) in series (1.4) together with Monte Carlo simulation was $67.31 \%$, but if we used both terms it was only $0.28 \%$. Over the runs we did for $\tau=0.5$, the maximum error in any run using only the constant term in (1.4) together with Monte Carlo was 100\% (the code returned a value of zero, when the actual value was nonzero), while if we used both terms, it was $0.53 \%$. However, for larger times ( $\tau$ between 2.23 and 3), the results were very poor, with an average error of $76.53 \%$ and maximum error of $100 \%$ using just the constant term, and an average error of $11.27 \%$ and a maximum error of $71.02 \%$ using both terms. In Figure 2.2, we present a subset of these results graphically. Figure 2.2a is for a constant boundary (i.e., the log term in (1.4) is absent), and it can be seen from the figure that these results are dreadful. We have included two separate figures for the case when the log term is present (Figures 2.2b and 2.2c). The first of these shows the behaviour for small tenor ( $T-t<1$ say), for which the approximation appears fairly good, although not as good as the call, while the second shows that the behaviour for larger tenor $(1<T-t<6$ here) is almost as bad as for the constant term. Earlier, we mentioned that the approximation (1.4) was only valid for $\tau \leq \sigma^{2} /\left(8 \pi\left(r-D_{0}\right)^{2}\right)$ because of the logarithm inside the square root, and it is as we approach this critical value that the approximation becomes very poor.

Finally, as we did for the call, in Tables 2.11 and 2.12, our results for the value of an option obtained using the series solution combined with Monte Carlo simulation are compared to previously published values obtained using other methods, specifically the LUBA approximation, the 


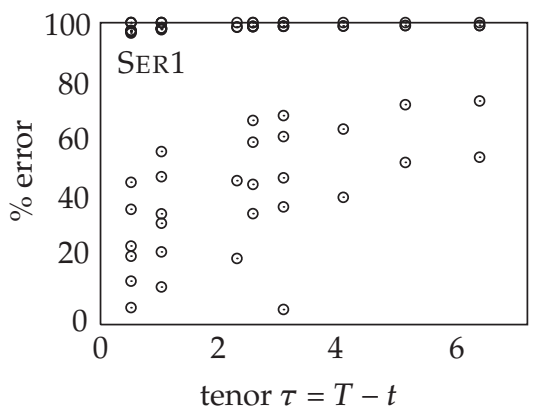

(a)

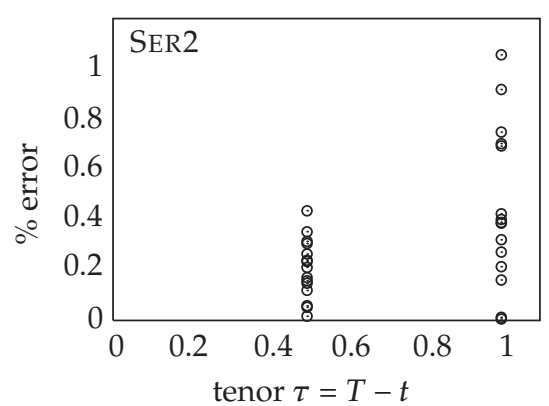

(b)

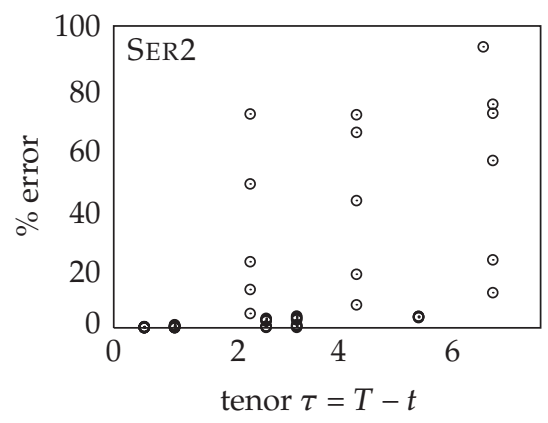

(c)

FIgURE 2.2. Percentage error in the value of a put option using series (1.4) for a number of sample runs. (a) constant term only; (b) and (c) both terms in series.

2-point Geske-Johnson approximation, the quadratic approximation and the method of lines. The parameter values chosen are the same as those used in earlier studies. We should mention that some of the results from other methods included in this table were originally presented in the literature for the call with $D_{0}>r$ and the results for the put with $D_{0}<r$ presented here were obtained using put-call symmetry (1.5). Paradoxically, the put series actually appears to be fairly good for the parameter values considered in Tables 2.11 and 2.12, although of course the scatter plots discussed above indicate that in many cases the truth is otherwise.

\section{Discussion}

In Section 2, we presented Monte Carlo simulations showing the return an investor holding an American option would expect if he used the 
88 Approximations for American options

TABle 2.11. Put: Comparison with other methods; $\tau=0.5, S_{0}=100$, $r=0.07, D_{0}=0.03$, and $\sigma=0.4$. SER is the value of an American option computed using using a 100,000 path Monte Carlo simulation taking series (1.4) as the exercise boundary. Other columns are as in Table 2.5.

\begin{tabular}{l|lllll}
\hline$E$ & 80 & 90 & 100 & 110 & 120 \\
\hline EURO. & 2.654 & 5.623 & 10.022 & 15.773 & 22.653 \\
AMER. & 2.689 & 5.722 & 10.239 & 16.181 & 23.360 \\
SER. & 2.694 & 5.714 & 10.214 & 16.156 & 23.305 \\
LUBA & 2.689 & 5.723 & 10.240 & 16.182 & 23.357 \\
GJ & 2.661 & 5.676 & 10.198 & 16.195 & 23.477 \\
QUAD. & 2.711 & 5.742 & 10.242 & 16.152 & 23.288 \\
ML & 2.668 & 5.715 & 10.241 & 16.187 & 23.370 \\
\hline
\end{tabular}

TABLE 2.12. Put: Comparison with other methods; as in Table 2.11 but $\tau=3.0$.

\begin{tabular}{l|lllll}
\hline$E$ & 80 & 90 & 100 & 110 & 120 \\
\hline EURO. & 10.309 & 14.162 & 18.532 & 23.363 & 28.598 \\
AMER. & 11.326 & 15.722 & 20.793 & 26.495 & 32.781 \\
SER7 & 11.302 & 15.653 & 20.664 & 26.306 & 32.439 \\
LUBA & 11.327 & 15.724 & 20.793 & 26.489 & 32.772 \\
GJ & 11.275 & 15.787 & 21.029 & 26.939 & 33.448 \\
QUAD. & 11.625 & 16.028 & 21.084 & 26.749 & 32.982 \\
ML & 11.278 & 15.683 & 20.752 & 26.464 & 32.756 \\
\hline
\end{tabular}

series approximation to the optimal exercise boundary as his exercise strategy. For the call, we found that using the series solution (1.3) would capture almost all of the values of an American call, even for large times, provided at least two terms in the series were used. Surprisingly, using more terms did not always guarantee more accuracy, as the results in Tables 2.1, 2.2, 2.3, and 2.4 attest. Of course, for such large times, it is questionable whether the Black-Scholes-Merton model is applicable, since it assumes constant volatility and constant interest rates, but our results for the call are none-the-less extremely encouraging. Because the agreement between the simulations and the values obtained using binomial trees is so good, an investor could use the series solution for the optimal exercise boundary as his exercise policy (as we have done in our simulations) and thereby be able to reap almost the entire early exercise premium of the call. The reason this is useful to an investor is that the series is so easy to evaluate that it could literally be programmed into a financial calculator and evaluated in fractions of a second, making it far more accessible to the average options investor than the large 
numerical codes often required to calculate the location of the boundary. As we noted in the introduction, over the years, a number of approximations have been proposed for the valuation of Americans and the calculation of the optimal exercise boundary, and the majority of these approximations are excellent. Our argument would be that series (1.3) is attractive because it is both reasonably accurate and also extremely easy to evaluate.

For the put, because of the log term in the approximation (1.4), the approximate location of the boundary can only be computed for comparatively small times, and we found that the asymptotic solution behaved well for very small times, but poorly, and in some cases very poorly, for times that were a little larger. Keeping more terms in the series would probably improve the performance of the boundary for the put, but there is presently no consensus as to the coefficients of the next terms in the series. Perhaps when the next few terms are available, it might be worthwhile to repeat the simulations for the put. Until that occurs, we would not recommend an investor use the series for the put, other than very close to expiry, because of its poor performance.

We turn now to the Greeks, meaning the sensitivity of the option's price to changes in the parameters or more precisely the derivatives of the option's price with respect to those parameters. Some of these are used extensively, for example $\Delta=\partial V / \partial S$ is used in hedging. Since the series solutions discussed here are approximations for the early exercise boundary rather than the value of the option, we must compute the Greeks numerically using central differences: for example, we have

$$
\begin{aligned}
& \Delta=\frac{\partial V}{\partial S}=\frac{V(S+\delta, t)-V(S-\delta, t)}{2 \delta}+\mathcal{O}\left(\delta^{2}\right) \\
& \Gamma=\frac{\partial^{2} V}{\partial S^{2}}=\frac{V(S+\delta, t)+V(S-\delta, t)-2 V(S, t)}{\delta^{2}}+\mathcal{O}\left(\delta^{2}\right)
\end{aligned}
$$

where $V(S, t), V(S+\delta, t)$, and $V(S-\delta, t)$ could be obtained by using Monte Carlo simulation as in Section 2, using series (1.3) and (1.4) as the optimal exercise boundary. As always when the Greeks are calculated in this way, their value will only be accurate if the value of the options in these formulae are accurate. For the call, the value of the Greeks should be pretty accurate, just as the value of the option was in Section 2.1. Conversely, since the series for the put behaved poorly in Section 2.2, except for very small times, we would expect the value of the Greeks to be rather inaccurate for the put. 


\section{References}

[1] F. Aitsahlia and T. L. Lai, Efficient approximations to American option prices, hedge parameters and exercise boundaries, Tech. Report 1998-24, Department of Statistics, Stanford University, October 1998.

[2] Exercise boundaries and efficient approximations to American option prices and hedge parameters and exercise boundaries, Tech. Report 2000-32, Department of Statistics, Stanford University, October 2000.

[3] G. Allegretto, G. Barone-Adesi, and R. J. Elliott, Numerical evaluation of the critical price and American options, The European Journal of Finance $\mathbf{1}$ (1995), 69-78.

[4] G. Alobaidi and R. Mallier, Asymptotic analysis of American call options, Int. J. Math. Math. Sci. 27 (2001), no. 3, 177-188.

[5] On the optimal exercise boundary for an American put option, J. Appl. Math. 1 (2001), no. 1, 39-45.

[6] G. Barles, J. Burdeau, M. Romano, and N. Samsoen, Critical stock price near expiration, Math. Finance 5 (1995), no. 2, 77-95.

[7] G. Barone-Adesi and R. J. Elliott, Approximations for the values of American options, Stochastic Anal. Appl. 9 (1991), no. 2, 115-131.

[8] G. Barone-Adesi and R. E. Whaley, Efficient analytic approximation of American option values, J. Finance 42 (1987), 301-320.

[9] P. Bjerksund and G. Stensland, Closed form approximation of American options, Scandinavian Journal of Management 9 (1993), 87-99.

[10] F. Black and M. Scholes, The pricing of options and corporate liabilities, Journal Political Economy 81 (1973), 637-654.

[11] E. C. Blomeyer, An analytic approximation for the American put price for options on stocks with dividends, J. Financial Quant. Anal. 21 (1986), no. 2, 229-233.

[12] P. Bossaert, Simulation estimators of optimal early exercise, Carnegie Mellon University, preprint, 1989.

[13] P. Boyle, Options: A Monte Carlo approach, J. Fin. Econ. 4 (1977), 323-338.

[14] A lattice framework for option pricing with two state variables, J. Fin. and Quant. Anal. 23 (1988), no. 1, 1-12.

[15] P. Boyle, A. W. Kolkiewicz, and K. S. Tan, Pricing American options using quasi-Monte Carlo methods, MCQMC 2000: 4th International Conference on Monte Carlo and Quasi-Monte Carlo Methods in Scientific Computing, Hong Kong, 2000.

[16] M. J. Brennan and E. S. Schwartz, The valuation of the American put option, J. Finance 32 (1977), no. 2, 449-462.

[17] M. Broadie and J. Detemple, American option valuation: new bounds, approximations, and a comparison of existing methods, Rev. Financ. Stud. 9 (1996), no. 4, 1211-1250.

[18] M. Broadie and P. Glasserman, Pricing American-style securities using simulation, J. Econom. Dynam. Control 21 (1997), no. 8-9, 1323-1352.

[19] D. S. Bunch and H. E. Johnson, A simple and numerically efficient valuation method for American puts using a modified Geske-Johnson approach, J. Finance 47 (1992), 809-816.

[20] , The American put option and its critical stock price, J. Finance 55 (2000), 2333-2356. 
[21] P. Carr, Randomization and the American put, Rev. Financ. Stud. 11 (1998), 597626.

[22] P. Carr and P. Faquet, Fast evaluation of American options, Working Paper, Cornell University, 1994.

[23] P. Carr, R. Jarrow, and R. Myneni, Alternative Characterizations of American put options, Math. Finance 2 (1992), 87-106.

[24] X. Chen and J. Chadam, Mathematical analysis for the optimal exercise boundary of American put option, University of Pittsburgh, preprint, 2001.

[25] X. Chen, J. Chadam, and R. Stamicar, The optimal exercise boundary for American put options: analytic and numerical aproximations, University of Pittsburgh, preprint, 2001.

[26] M. Chesney and R. Gibson, State space symmetry and two factor option pricing models, Applied Stochastic Models and Data Analysis, Vol. I, II (Chania, 1993), World Scientific Publishing, New Jersey, 1993, pp. 136-160.

[27] J. Cox, S. Ross, and M. Rubinstein, Option pricing: a simplified approach, J. Fin. Econ. 7 (1979), 229-264.

[28] J. N. Dewynne, S. D. Howison, I. Rupf, and P. Wilmott, Some mathematical results in the pricing of American options, European J. Appl. Math. 4 (1993), no. 4, 381-398.

[29] J. D. Evans, R. E. Kuske, and J. B. Keller, American options with dividends near expiry, preprint, 2001.

[30] B. Gao, J. Huang, and M. Subrahmanyam, The valuation of American barrier options using the decomposition technique, J. Econom. Dynam. Control 24 (2000), no. 11-12, 1783-1827.

[31] R. Geske, A note on an analytical valuation formula for unprotected American call options on stocks with known dividends, J. Financial Econ. 7 (1979), 375-380.

[32] Comments on Whaley's note, J. Financial Econ. 9 (1981), 213-215.

[33] R. Geske and H. E. Johnson, The American put valued analytically, J. Finance 39 (1984), 1511-1524.

[34] R. Geske and K. Shastri, Valuation by approximation: a comparison of alternative option valuation techniques, J. Financial Quant. Anal. 20 (1985), 45-71.

[35] J. Huang, M. Subrahmanyam, and G. Yu, Pricing and hedging American options: a recursive integration method, Rev. Financ. Stud. 9 (1996), 277-300.

[36] A. Ibanez and F. Zapatero, Monte Carlo Valuation of American Options through Computation of the Optimal Exercise Frontier, 99-8, Marshall School of Business, University of Southern California, preprint, 1999.

[37] H. E. Johnson, An analytical approximation to the American put price, J. Financial Quant. Anal. 18 (1983), 141-148.

[38] N. Ju, Pricing an American option by approximating its early exercise boundary as a multipiece exponential function, Rev. Financ. Stud. 11 (1998), 627-646.

[39] R. E. Kuske and J. B. Keller, Optimal exercise boundary for an American put option, Appl. Math. Fin. 5 (1998), 107-116.

[40] P. L'Ecuyer and S. Côté, Implementing a random number package with splitting facilities, ACM Trans. Math. Software 17 (1991), no. 1, 98-111.

[41] L. W. MacMillan, Analytic approximation for the American put option, Advances in Futures and Options Research 1A (1986), 119-139.

[42] R. Mallier, Approximating the optimal exercise boundary for American options via Monte Carlo, Computational Intelligence: Methods and Applications 


\section{Approximations for American options}

(CIMA2001) (L. I. Kuncheva et al., eds.), ICSC Academic Press, Canada, 2001, pp. 365-371.

[43] R. Mallier and G. Alobaidi, Using Monte Carlo methods to evaluate sub-optimal exercise policies for American options, MCQMC 2000: 4th International Conference on Monte Carlo and Quasi-Monte Carlo Methods in Scientific Computing, Hong Kong, 2000.

[44] R. McDonald and M. Scroder, A parity result for American options, J. Comp. Fin 1 (1998), 5-13.

[45] W. Melick and C. Thomas, Recovering an asset's implied pdf from option prices: an application to crude oil during the Gulf war, J. Finan 32 (1997), 91-115.

[46] R. C. Merton, The theory of rational option pricing, Bell J. Econ. and Management Science 4 (1973), 141-183.

[47] On the problem of corporate debt: the risk structure of interest rates, J. Finance 29 (1974), 449-470.

[48] R. Roll, An analytical formula for unprotected American call options on stocks with known dividends, J. Financial Econ. 5 (1977), 251-258.

[49] M. J. P. Selby and S. D. Hodges, On the evaluation of compound options, Management Science 33 (1987), 347-355.

[50] R. Stamicar, D. Sevcovic, and J. Chadam, The early exercise boundary for the American put near expiry: numerical approximation, Canad. Appl. Math. Quart. 7 (1999), 427-444.

[51] J. Tilley, Valuing American options in a path simulation model, Trans. Soc. Actuaries 45 (1983), 83-104.

[52] R. E. Whaley, On the valuation of American call options on stocks with known dividends, J. Financial Econ. 9 (1981), 207-211.

[53] P. Wilmott, Derivatives. The Theory and Practice of Financial Engineering, Wiley \& Sons, Chichester, 1998.

[54] L. Wu and Y.-K. Kwok, A front-fixing finite difference method for the valuation of American options, J. Financial Engineering 6 (1997), 83-97.

Roland Mallier: Department of Applied Mathematics, University of Western Ontario, London, ON, Canada N6A 5B7

E-mail address: mallier@uwo.ca 


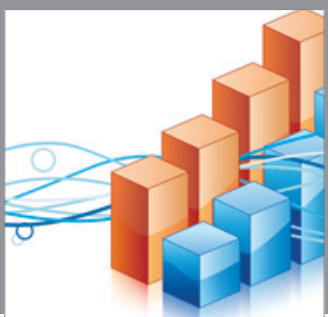

Advances in

Operations Research

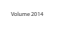

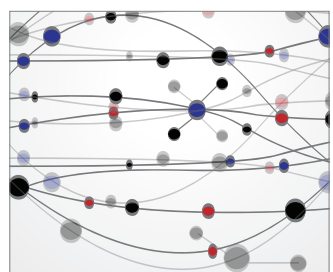

\section{The Scientific} World Journal
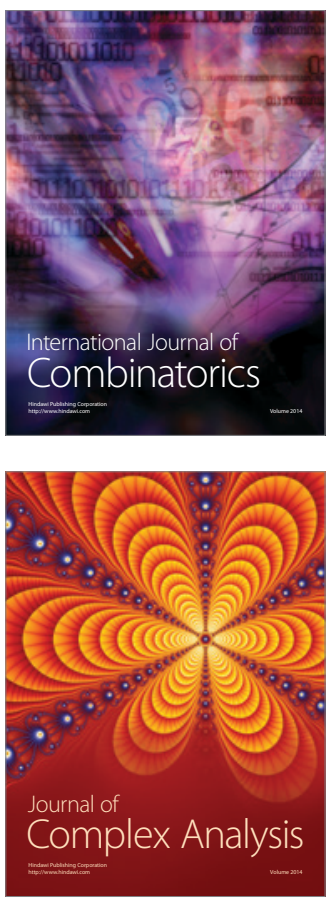

International Journal of

Mathematics and

Mathematical

Sciences
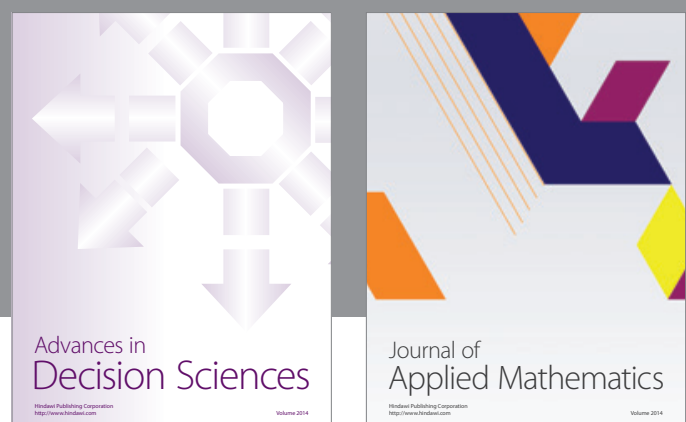

Journal of

Applied Mathematics
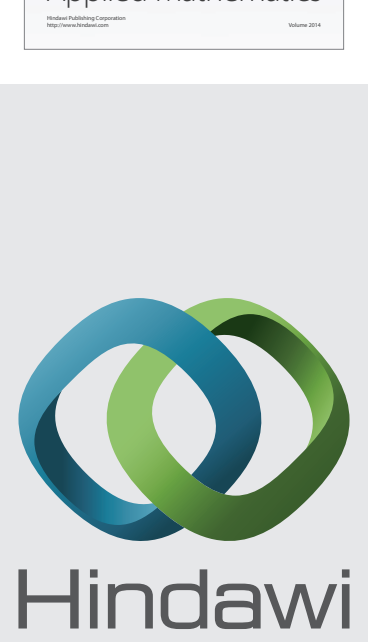

Submit your manuscripts at http://www.hindawi.com
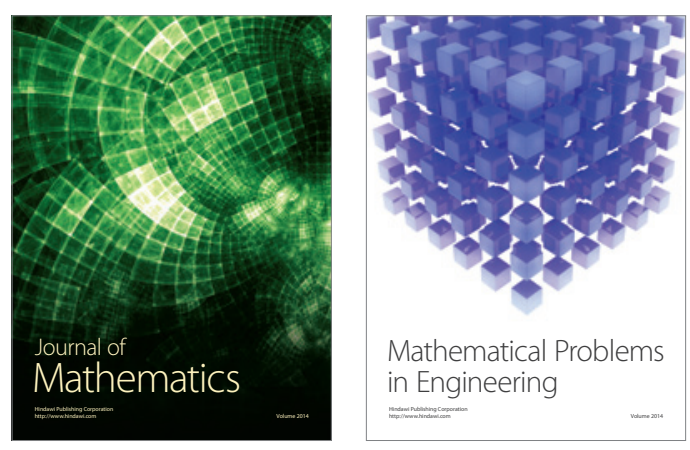

Mathematical Problems in Engineering
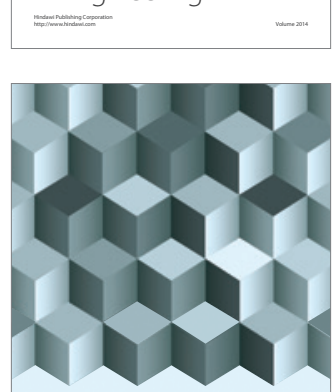

Journal of

Function Spaces
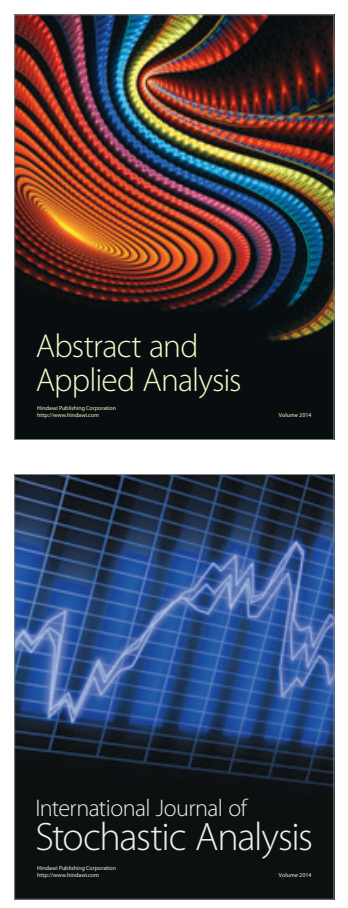

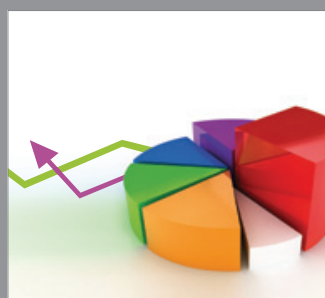

ournal of

Probability and Statistics

Promensencen
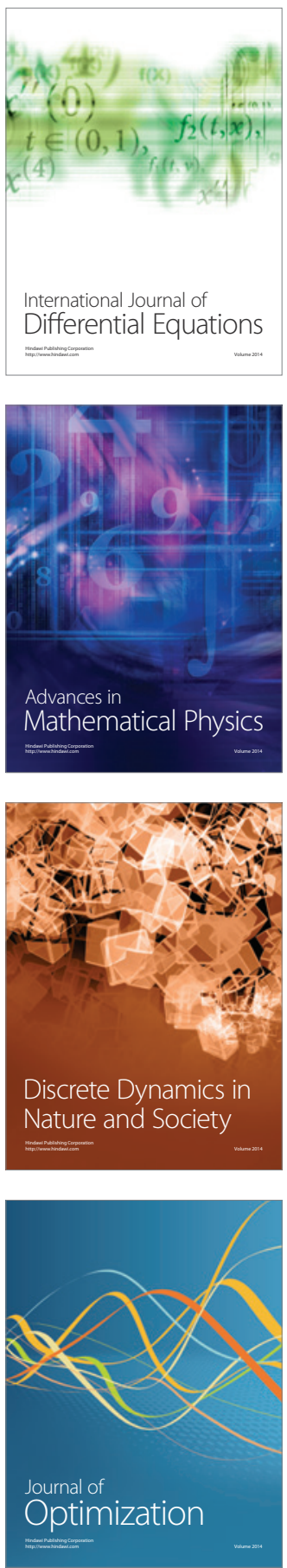